Cahiers de recherches médiévales

\title{
César et les Bretons
}

Les premiers pas des Romains en Grande Bretagne dans l'historiographie latine, de César à Geoffroy de Monmouth

\section{Silvère Menegaldo}

\section{(2) OpenEdition}

\section{Journals}

Édition électronique

URL : https://journals.openedition.org/crm/2557

DOI : $10.4000 / \mathrm{crm} .2557$

ISSN : $1955-2424$

Éditeur

Honoré Champion

Édition imprimée

Date de publication : 30 juin 2007

Pagination : $29-41$

ISSN : 1272-9752

Référence électronique

Silvère Menegaldo, "César et les Bretons », Cahiers de recherches médiévales [En ligne], 14 spécial |

2007, mis en ligne le 30 juin 2010, consulté le 15 décembre 2022. URL : http://

journals.openedition.org/crm/2557 ; DOI : https://doi.org/10.4000/crm.2557 


\title{
RM
}

\author{
César et les Bretons. \\ Les premiers pas des Romains en Grande Bretagne dans \\ l'historiographie latine, de César à Geoffroy de Monmouth
}

D'emblée disons que je voudrais aborder cette question d'histoire dans une perspective un peu particulière, et pour la préciser il me faut revenir en arrière, au colloque qui a précédé celui-ci, où je m'étais intéressé à un roman en vers assez peu connu, le Roman de Jules César de Jean de Thuin, adaptation plutôt que traduction de La Guerre civile de Lucain probablement composée vers $1260^{1}$. À cette occasion je m'étais posé la question suivante: pourquoi a-t-il fallu attendre aussi longtemps pour qu'un auteur se décide à adapter Lucain, dont le texte a toujours été très répandu au Moyen Âge, alors que dès la seconde moitié du XII ${ }^{\mathrm{e}}$ siècle, entre 1150 et 1165, sont successivement transposés en français la Thébaïde de Stace, avec le Roman de Thèbes, L'Énéide de Virgile, avec le Roman d'Eneas, et les résumés de la guerre de Troie composés par Darès et Dictys, avec le Roman de Troie de Benoît de Sainte-Maure ? Parmi diverses suggestions d'explication - ainsi l'inachèvement de La Guerre civile et la difficulté d'accès des textes à même de la compléter, ou bien le statut problématique du personnage de César dans le poème de Lucain - se trouvait encore la suivante, directement liée au contexte de production de ces œuvres : il semble en effet avéré ${ }^{2}$ que ces «romans antiques», comme on les appelle, ont tous trois été composés dans le même milieu, celui de la cour d'Henri II Plantagenêt, et dans la même perspective, celle de doter la dynastie anglo-angevine de prestigieuses origines remontant aux héros troyens, perspective dont témoigne notamment l'association fréquente dans les manuscrits de ces trois romans et de textes, comme le Roman de Brut de Wace, consacrés à l'histoire de la Grande Bretagne et à ses glorieuses figures, bretonnes d'abord, puis normandes ; or dans une telle perspective, où ce sont les Bretons - et le premier d'entre eux, Arthur - et non les Saxons qui se trouvent valorisés comme les prestigieux ancêtres des Normands, Jules César, le vainqueur des Gaules et de la Bretagne, apparaît nécessairement sous le visage de l'envahisseur et de l'ennemi.

C'est en tout cas ce qui se manifeste particulièrement chez un historiographe étroitement lié au milieu normand, Geoffroy de Monmouth, auteur de l'Historia regum Britannia (vers 1138), qui dans son désir de glorifier les Bretons va jusqu'à en faire les égaux, et même les supérieurs des Romains, la victoire de ces derniers sur les premiers, que Geoffroy ne peut tout de même nier, se trouvant en effet, dans l'ensemble de l'Historia, doublement contrebalancée: d'abord, bien avant les expéditions césariennes, quand Belin et Brenne prennent Rome (voir $\S 43$ ), et ensuite quand Arthur, bien plus tard, manque de renouveler le même exploit (voir

\footnotetext{
${ }^{1}$ «César 'd'ire enflamez et espris' (v. 1696) dans Le Roman de Jules César de Jean de Thuin », Cahiers de Recherches Médiévales, numéro 13 spécial sur «La figure de Jules César au Moyen Âge et à la Renaissance », 2006, p. 59-76.

${ }^{2}$ Voir notamment la mise au point de M. Aurell dans L'Empire des Plantagenêt, Paris, Perrin, 2004, p. 173-174.
}

Cahiers de Recherches Médiévales, 14spé, 2007 
$\S 176$ ). En outre dans l'Historia les premiers pas de Jules César en Bretagne (§ 5463) donnent des Romains une image particulièrement peu flatteuse, aussi bien au regard de leur comportement cupide et plein de morgue, contrastant avec la fierté et l'indépendance des Bretons, que de la succession de défaites humiliantes qu'ils doivent subir, sans jamais remporter de véritable victoire. Cette vision des événements apparaît d'ailleurs tellement défavorable aux Romains qu'elle se trouvera rapidement remise en cause: violemment critiquée par un historien contemporain de Geoffroy, Guillaume de Newburgh ${ }^{3}$, elle sera aussi sensiblement corrigée par son traducteur en français, puisque Wace, dans son Roman de Brut, s'emploiera par divers moyens à redorer le blason césarien, dont le plus évident apparaît le portrait très élogieux qu'il propose d'emblée, avant de relater son expédition bretonne, de «Julius Cesar li vaillanz / Li forz, li pruz, li conqueranz $»^{4}$.

La question se pose alors de savoir s'il a existé, en dehors du seul Geoffroy, une tradition spécifiquement bretonne concernant l'histoire des premiers pas romains en Grande Bretagne, à laquelle serait liée une vision négative du général romain, et dans ce cas comment cette tradition a pu se construire face à une tradition romaine évidemment bien différente et beaucoup plus favorable aux Romains, comme on va le voir immédiatement avec le récit que fait César lui-même de ses campagnes bretonnes ${ }^{5}$ ?

\footnotetext{
${ }^{3}$ Guillaume de Newburgh fait dans le prologue de son Historia rerum anglicarum une critique en règle de toutes les inventions et de tous les mensonges que contient selon lui l'œuvre de son devancier Geoffroy de Monmouth, et dans un des passages les plus virulents de cette critique, il renvoie sinon directement du moins allusivement à l'expédition césarienne en Bretagne: Praterea in libro suo, quem Britonum historiam vocat, quam petulanter et quam impudenter fere per omnia mentiatur nemo nisi veterum historiarum ignarus, cum in librum illum inciderit, ambigere sinitur. Nam qui rerum gestarum veritatem non didicit fabularum vanitatem indiscrete admittit. Omitto quanta de gestis Britonum ante Julii Casaris imperium homo ille confinxerit, vel ab aliis conficta tanquam authentica scripserit. Omitto quacunque in laudibus Britonum contra fidem historica veritatis deliravit, a tempore Julii Casaris, sub quo Britones ditionis Romance esse coeperunt, usque ad tempus Honorii imperatoris, sub quo Romani a Britannia propter urgentiora reipublica negotia sponte recesserunt (Guillaume de Newburgh, Historia rerum anglicarum, éd. et trad. P.G. Walsh et M.J. Kennedy, Warminster, Aris and Phillips, 1988, p. 30 ; c'est moi qui souligne).

${ }^{4}$ Wace, Roman de Brut, éd. et trad. J. Weiss, University of Exeter, 1999, v. 3833 et suivants. Sur le traitement que propose Wace de cet épisode, voir notamment F.H.M. Le Saux, $A$ Companion to Wace, Cambridge, Brewer, 2005, p. 114-115, et C. Croizy-Naquet, «César et le romanz au XII ${ }^{\mathrm{e}}$ siècle», Cahiers de Recherches Médiévales, numéro 13 spécial sur «La figure de Jules César au Moyen Âge et à la Renaissance », 2006, p. 39-49.

${ }^{5}$ Cette question est déjà abordée dans l'ouvrage de J. Leeker, Die Darstellung Cäsars in den romanischen Literaturen des Mittelalters (Francfort, Klostermann, 1986, p. 172-185), dont les développements sont eux-mêmes fortement redevables à l'article de H. Nearing, "The Legend of Julius Caesar's British Conquest», Publications of the Modern Language Association of America, 64, 1949, p. 889-929. Sans prétendre la renouveler complètement, nous nous sommes au moins efforcés d'ajouter à cette étude déjà ancienne quelques références (Tite-Live, Plutarque, Florus), ainsi que des éléments d'interprétation sur le déconcertant parcours historiographique des expéditions romaines en Bretagne.
} 


\section{La période romaine: César et ses juges}

Nous allons donc aborder le récit des premiers pas romains en Bretagne, en commençant bien sûr par César lui-même, qui propose une relation détaillée de ses deux expéditions bretonnes dans le De bello gallico (livre IV, 20-38 et livre V, 1$23)^{6}$. Le récit de César est, sinon la seule, du moins la source première et essentielle à même de nous faire connaître les événements qui ont effectivement eu lieu lors de ces expéditions; on peut donc le suivre pour l'établissement du fait historique, ce que je ferai ici, en m'appuyant également sur le résumé condensé, et éclairé par quelques autres sources, qu'en propose l'historien Y. Le Bohec ${ }^{7}$.

La première expédition de Bretagne se trouve relatée dans le livre IV du $D e$ bello gallico. Passons d'emblée sur les raisons mêmes de l'expédition, qui n'apparaissent pas évidentes : en tout cas il ne s'agissait certainement pas d'envahir la Bretagne, étant donné le peu d'effectifs des troupes romaines, mais plutôt d'effectuer une reconnaissance et d'accroître symboliquement la gloire césarienne en franchissant l'Océan pour aborder dans un pays rendu attirant par le mystère et les richesses qu'il recelait peut-être. Après divers préparatifs - dont l'envoi d'une mission de reconnaissance romaine et d'une ambassade gauloise pour engager les Bretons à se soumettre - César embarque fin août 55, probablement de Boulogne, avec une centaine de vaisseaux transportant deux légions ainsi que de la cavalerie. Il atteint la côte anglaise du côté de Douvres, mais renonce à débarquer, le rivage étant couvert d'ennemis en armes ; finalement le débarquement s'effectue, au terme d'un combat difficile entre mer et plage, un peu plus au Nord. Le premier affrontement entre Bretons et Romains est donc en faveur de ces derniers et suite à cette première défaite les Bretons viennent demander la paix et livrent des otages. Cependant, quatre jours après, une violente tempête prive César de sa cavalerie, qui a quitté plus tard le continent, et endommage une partie de ses navires. Voyant les Romains en difficulté, les Bretons interrompent la livraison des otages, tendent une embuscade à des soldats partis récolter du blé (c'est là que prend place un développement de César sur les chars bretons, au $\S 33$ ), puis attaquent le camp romain, mais prennent la fuite face aux légions en ordre de bataille. Après de nouvelles demandes de paix et la promesse de livrer des otages, César s'en retourne sur le continent à la fin du mois de septembre.

Quant à la seconde expédition de Bretagne, qui eut lieu l'année suivante (en 54), elle est relatée à la suite de la première au début du livre V du De bello gallico. Les otages promis n'ayant pas été livrés, c'est du moins le prétexte, César lance une seconde expédition de plus d'ampleur, avec environ 800 navires, cinq légions et 2000 cavaliers, pour beaucoup des Gaulois que le général romain emmène avec leurs chefs pour éviter qu'ils ne se révoltent. L'embarquement, retardé par une série de contretemps, a lieu certainement au même endroit que la première fois, à Boulogne, début août; et le débarquement s'effectue également au même endroit, mais beaucoup plus facilement que le premier, les Bretons ayant été selon César «effrayés à la vue de tant de vaisseaux» (\$ 8). Tandis que les Romains dressent leur

${ }^{6}$ César, La Guerre des Gaules, éd. et trad. L.-A. Constans, Paris, Les Belles Lettres, 1926, deux tomes.

${ }^{7}$ Y. Le Bohec, César chef de guerre, Paris, Le Rocher, 2001, p. 220-230. 
camp, une partie de l'armée commence à entrer dans les terres et repousse les Bretons, qui se cachent dans des sortes de bois fortifiés ( $\$ 9$ ). Mais voici, exactement comme lors de la première expédition, que se déchaîne une nouvelle tempête : les Romains, qui perdent 40 navires, sont contraints de tirer tous les autres au sec et de les enfermer dans l'enceinte de leur camp. De leur côté les Bretons se rassemblent sous le commandement d'un certain Cassivellaunos. À ce moment-là César intercale une description de la Bretagne ( $\S 12-14)$. Puis ont lieu divers combats, au cours desquels les Romains ont «partout le dessus » (§ 15), en dépit de quelques pertes (dont celle du tribun militaire Q. Laberius) et des difficultés tactiques posées par l'emploi de la cavalerie ; cependant cette série d'affrontements se solde par une franche victoire romaine, et la déroute des Bretons. Alors les Romains continuent leur avancée et franchissent la Tamise à l'Ouest de Londres, en dépit des pieux enfoncés dans les berges et le lit du fleuve ( $§ 18)$. Les Bretons, toujours sous les ordres de Cassivellaunos, renoncent à la bataille rangée et pratiquent une tactique de guérilla, à laquelle les Romains répondent par une politique de terre brûlée. Mais finalement un certain Mandubracios, prince des Trinovantes, dont le père a été tué par Cassivellaunos, vient se soumettre à César, et d'autres peuples après lui, si bien qu'isolé et encore vaincu à plusieurs reprises, Cassivellaunos se soumet à son tour et promet de verser un tribut à Rome. César rejoint le continent à la fin du mois de septembre : la seconde expédition a ainsi duré à peu près deux fois plus longtemps que la première et a conduit César assez avant dans le pays, au-delà de Londres.

Voilà donc pour les faits, étant donné que d'un point de vue factuel le récit de César reste la source primordiale et une source difficilement discutable; en effet on ne dispose par ailleurs chez les historiens antiques que de textes résumant ces mêmes événements sans leur apporter grand-chose de bien nouveau. Reste cependant ce qui concerne l'évaluation de ces faits, c'est-à-dire le succès de ces deux expéditions, dont les historiens discutent encore aujourd'hui, et discutaient déjà dès l'époque romaine.

Évidemment César lui-même présente de façon très favorable les deux expéditions qu'il a menées: tous les affrontements tournent à l'avantage des Romains, en dépit de certaines difficultés tactiques (le premier débarquement, les chars bretons, l'usage de la cavalerie); en outre, paradoxalement, ce sont plutôt les Bretons que les Romains qui sont présentés comme les agresseurs (voir en particulier le $\S 27$ du livre IV, où César reproche aux Bretons «de lui avoir fait la guerre sans motif»); les revers romains sont d'abord dus aux caprices de la nature, et spécialement aux tempêtes, plutôt qu'à leurs adversaires; les Bretons sont finalement soumis; enfin César conclut son libre IV du De bello gallico en notant qu'en l'honneur de ses exceptionnels succès, soit le franchissement du Rhin et de l'Océan, le Sénat «décréta vingt jours d'actions de grâces » (§ 38). Et environ deux cents ans plus tard, c'est le même bilan extrêmement flatteur qui se trouve rapporté dans un texte à dire vrai plus soucieux de rhétorique que de vérité historique, l'abrégé d'histoire romaine de Florus (livre $\mathrm{I}, 45,18$ ), où il n'est question que de la «rapidité » et de l'«audace» de César, que de la «panique» des Bretons, incapables de s'opposer aux Romains, et qui conclut ainsi, à propos du général romain : «se

${ }^{8}$ Florus, Euvres, éd. et trad. P. Jal, Paris, Les Belles Lettres, 1967, tome I. 
contentant de cette victoire - ce n'était pas la province, mais la gloire qui l'intéressait - il revint avec un butin plus grand que précédemment ${ }^{9}$; l'Océan lui aussi fut plus tranquille et plus propice pendant sa traversée, comme s'il reconnaissait son infériorité ».

Certains autres jugements sont en revanche plus mitigés, voire franchement défavorables. Ainsi Tite-Live adopte-t-il une position en quelque sorte médiane, en faisant de la première expédition un échec, et de la seconde un succès; c'est du moins l'avis que l'on trouve exprimé dans l'abrégé du livre 105 de l'Histoire romaine, qui ne nous est pas parvenu en intégralité : César, «par l'Océan, [...] passa en Bretagne, sans obtenir, la première fois, beaucoup de succès, en raison de l'obstacle des tempêtes; une deuxième fois, avec plus de chance; et, après avoir massacré un très grand nombre d'ennemis, il fit passer en son pouvoir une notable partie de l'île ${ }^{10}$. De son côté Tacite, dans la Vie d'Agricola $(13,2)^{11}$, ne nie pas la nouveauté de l'exploit, mais en relativise l'importance: «le premier de tous les Romains, le divin César entra en Bretagne avec une armée ; par un combat heureux il effraya les habitants et s'empara de la côte ; mais on peut juger qu'il montra cette conquête à ses successeurs plutôt qu'il ne la leur transmit». On trouve encore des idées similaires, soulignant à la fois l'audace de l'entreprise et son peu de résultats, dans la Vie de César de Plutarque $(\$ 23)^{12}$. De même, pour Dion Cassius, César n’a rien gagné sinon la gloire d'avoir conduit cette expédition, et là-dessus sa fierté est grande $^{13}$. Mais c'est à Lucain que revient de porter le jugement de loin le plus défavorable, voire franchement calomnieux, mais il est vrai qu'il est mis dans la bouche de Pompée, se demandant d'où vient l'audace de César: «Est-ce pour avoir fui loin des eaux glacées du Rhin et, appelant Océan les bas-fonds d'une mer incertaine, montré un dos effrayé aux Bretons qu'il était venu chercher?» (livre II, v. $570-572)^{14}$.

Tel est donc, à l'exception d'un auteur sur lequel nous allons très bientôt revenir, le bilan de l'historiographie antique concernant les expéditions bretonnes de César, où l'on peut distinguer d'un côté un récit proprement événementiel dont la source essentielle, sinon unique, est le De bello gallico, sur lequel très certainement tous les historiens postérieurs s'appuient, et de l'autre un ensemble de jugements, plus ou moins favorables, sur le succès de ces expéditions. Qu'en retrouve-t-on au Moyen Âge?

\footnotetext{
${ }^{9}$ Noter qu'il n'a jamais été question de butin dans la relation de César; le désir de glorification apparaît d'autant plus évident.

${ }^{10}$ Tite-Live, Abrégés des Livres de l'Histoire romaine, éd. et trad. P. Jal, Paris, Les Belles Lettres, 1984, tome deux, p. 32.

${ }^{11}$ Tacite, Vie d'Agricola, éd. et trad. E. de Saint-Denis, Paris, Les Belles Lettres, 1942.

${ }^{12}$ Plutarque, Vies parallèles, Paris, GF-Flammarion, 1995, tome un, p. 146-147

${ }^{13}$ Dion Cassius, Histoire romaine, livre XXXIX, 53. Cette partie de l'œuvre, où se lit le récit de la première expédition bretonne (livre XXXIX, 50-53), est notamment accessible en traduction anglaise dans la Loeb Classical Library. En revanche le récit de la seconde expédition (livre XL, 1-4) peut se lire dans la traduction française de M. Rossellini (Paris, Les Belles Lettres, 1996).

${ }^{14}$ Lucain, La Guerre civile, éd. et trad. A. Bourgery et M. Ponchon, Paris, Les Belles Lettres, 1927-1930.
} 


\section{Le haut Moyen Âge : Bède le Vénérable et l'Historia Brittonum}

Le premier jalon à considérer, dans l'historiographie médiévale insulaire, est évidemment l'Histoire ecclésiastique du peuple anglais de Bède le Vénérable, publiée en 731. C'est, pour ce qui nous concerne, le premier texte auquel nous arrêter, puisque le De excidio et conquestu Britannia de Gildas (que l'on peut dater des environs de 550) ne dit rien de César ${ }^{15}$.

Bède consacre donc le second chapitre de son premier livre, juste après la description de la Grande Bretagne, à l'arrivée de César dans l'île, qui constitue le début de son histoire proprement dite. Je cite ce texte important dans son intégralité :

Comment Jules César fut le premier parmi les Romains à pénétrer en Bretagne

En vérité, cette Bretagne resta, pour les Romains, jusqu'à Jules César, inaccessible et inconnue. En la $693^{\mathrm{e}}$ année après la fondation de Rome, la $60^{\mathrm{e}}$ avant l'incarnation du Seigneur, César, qui venait de remplir son mandat de consul avec Lucius Bibulus, au cours de la guerre qu'il menait contre les peuples germains et gaulois, qui n'étaient séparés que par le Rhin, parvint chez les Morins, d'où la traversée vers la Bretagne est la plus courte et la plus brève ; après avoir équipé des vaisseaux légers et des transports, environ quatre-vingts, il passe en Bretagne ; là, d'abord éprouvé par une rude bataille, essuyant ensuite une terrible tempête, il perdit la plus grande partie de la flotte, un nombre importante de soldats, et presque la totalité de ses cavaliers. Revenu en Gaule, il envoya les légions dans leurs quartiers d'hiver et fit construire six cents navires des deux types.

Avec ceux-ci, il passa de nouveau en Bretagne, au début du printemps ; tandis qu'il marchait contre l'ennemi avec son armée, les navires, qui étaient à l'ancre, subirent une tempête et furent endommagés, soit parce qu'ils se heurtaient les uns contre les autres, soit parce qu'ils étaient enlisés dans les sables et disloqués ; quarante furent détruits tandis que le reste fut réparé au prix de grandes difficultés. Lors d'un premier engagement, la cavalerie de César fut vaincue par les Brittons et c'est alors que fut tué le tribun Labienus. Dans un second combat, au prix de grands risques pour les siens, il triompha des Brittons et les mit en fuite, et, de là, il se dirigea vers la Tamise. Sur la rive opposée, avait pris position, sous le commandement de Cassobellaunus, une foule immense d'ennemis qui avaient fiché, sur la rive du fleuve et sous l'eau, dans presque tout le gué, des pieux très acérés : les traces de ces pieux y sont encore visibles aujourd'hui, et l'observation révèle que chaque pieu avait la grosseur d'un fémur humain, et qu'après avoir été recouvert de plomb, il était solidement enfoncé dans le lit du fleuve.

Les Romains découvrirent ce traquenard et l'évitèrent; les barbares, ne pouvant endurer le choc des légions, se cachèrent dans les forêts, d'où ils faisaient de fréquentes sorties, en infligeant aux Romains de graves et fréquents dommages. Cependant les Trinovantes, une nation très puissante, se soumirent à César avec leur chef Andragius, en livrant quarante otages; plusieurs autres villes, ayant suivi cet

${ }^{15}$ Gildas, The Ruin of Britain and Other Works, éd. et trad. M. Winterbottom, Londres et Chichester, Phillimore, 1978. Le De excidio peut se lire aussi dans la traduction française de C. Kerboul-Vilhon, Sautron, Éditions du Pontig, 1996. Dans ce texte Gildas évoque bien la conquête romaine (§ 5-7), mais de façon générale, sans parler spécialement de César. 
exemple, conclurent une alliance avec les Romains. Grâce à leurs indications, César finit par s'emparer, au prix d'une dure bataille, de la place forte de Cassobellaunus, située entre deux marécages, défendue par un rideau de forêts et remplie de provisions de toute sorte. Ensuite César, revenu de Bretagne en Gaule, après avoir envoyé ses légions dans leurs quartiers d'hiver, fut subitement entouré de toutes parts par des soulèvements imprévus, et dut y faire face. ${ }^{16}$

À la lecture de ce texte, que constate-t-on? D'abord plusieurs erreurs ou imprécisions, concernant les dates (la première expédition est datée de 60 avant Jésus-Christ ; la seconde a lieu «au début du printemps»), les données chiffrées (80 navires pour la première expédition, au lieu d'une centaine ; 600 pour la seconde, au lieu de 800), ou encore certains noms (le tribun militaire tué lors de la seconde expédition est appelé Labienus au lieu de Laberius; le chef breton Cassobellaunus au lieu de Cassivellaunos; le chef des Trinovantes Andragius au lieu de Mandubracios). Ensuite et surtout le récit, fortement condensé, apparaît bien moins favorable aux Romains. Le résumé de la première campagne - où César, «d'abord éprouvé par une rude bataille, essuyant ensuite une terrible tempête, [...] perdit la plus grande partie de la flotte, un nombre importante de soldats, et presque la totalité de ses cavaliers »- n'a que bien peu à voir avec la relation du De bello gallico. De même lors de la seconde campagne, Bède souligne à plusieurs reprises les échecs des Romains : «lors d'un premier engagement, la cavalerie de César fut vaincue par les Brittons et c'est alors que fut tué le tribun Labienus », alors que César dans son récit parle seulement de ses difficultés à utiliser la cavalerie $(\mathrm{V}, 15-16)$; les Bretons se cachent dans les forêts «d'où ils faisaient de fréquentes sorties, en infligeant aux Romains de graves et fréquents dommages »; enfin la place forte de Cassobellaunus est prise «au prix d'une dure bataille », là où César parle d'une "courte résistance » $(\mathrm{V}, 21)$.

Même si Bède est anglais d'origine, et non breton, on pourrait être tenté de voir l'expression d'une sorte de chauvinisme dans cette présentation assez nettement défavorable des expéditions césariennes. Or il n'en est probablement rien en fait, puisque le récit de Bède n'a pas été fait d'après César, mais reprend mot pour mot ou presque, et erreur pour erreur, celui que propose Orose dans ses Histoires (livre VI, 9, 2-9) ${ }^{17}$. Sachant cependant, selon M.-P. Arnaud-Lindet, que César constitue bien la source principale du récit d'Orose ${ }^{18}$, la question se pose alors de savoir comment et pourquoi le compilateur chrétien a pu produire un tel résumé des expéditions césariennes. La réponse, qui ne semble faire guère de doute, tient en fait à la façon même dont Orose a dû travailler, et aux intentions qui l'animaient ${ }^{19}$. En premier lieu, Orose a travaillé très vite, puisque les sept livres des Histoires sont rédigés en l'espace de deux ans (416-417), et ses sources ont été rapidement

\footnotetext{
${ }^{16}$ Bède le Vénérable, Histoire ecclésiastique du peuple anglais, éd. de M. Lapidge, trad. de P. Monat et P. Robin, introduction et notes d'A. Crépin, Paris, Le Cerf (Sources chrétiennes 489, 490 et 491), 2005, volume un, p. 121-125.

${ }^{17}$ Voir Orose, Histoires, éd. et trad. M.-P. Arnaud-Lindet, Paris, Les Belles Lettres, 19901991, tome deux, p. 188-189.

${ }^{18}$ Ibid., p. 263.

${ }^{19}$ Nous suivons l'introduction de M.-P. Arnaud-Lindet à son édition.
} 
parcourues, parfois même, comme le note M.-P. Arnaud-Lindet précisément à propos du De bello gallico, avec «hâte» et «manque de réflexion $»^{20}$; il est donc probable que la plupart des erreurs commises par Orose trouvent ainsi leur explication, qu'il s'agisse des erreurs de chiffres (en fait Orose mentionne systématiquement, concernant le nombre de navires, le premier chiffre donné par César, sans tenir compte des précisions qu'il ajoute ensuite) ou de noms (ainsi la substitution à Laberius de Labienus s'explique par la fréquence d'apparition de ce nom dans le De bello gallico, qui désigne un légat de l'armée césarienne) $)^{21}$. Quant à la tournure nettement défavorable à César que prend le récit des deux expéditions, plutôt qu'à la hâte, elle est probablement à imputer aux intentions mêmes d'Orose : au départ, en effet, la rédaction des Histoires devait répondre au besoin de saint Augustion d'une «liste de catastrophes » pour servir d'arguments à opposer aux païens dans la rédaction des livres IV et V de La Cité de Dieu, des arguments notamment à même de montrer que la fin de la République n'avait pas été l'époque la plus glorieuse de Rome, et donc de dissuader de voir la moindre coïncidence entre la naissance du christianisme et la décadence romaine. Or même une fois que saint Augustin eut renoncé à se servir d'une telle liste, cette perspective d'apologétique chrétienne n'en est pas moins restée très visible dans la rédaction définitive des Histoires ; ainsi, comme le note encore M.-P. Arnaud-Lindet, «voulant [...] mettre en évidence le fait que, à partir de la naissance du Christ, les malheurs du monde ont graduellement diminué en fréquence et en intensité, Orose doit établir que les deux derniers siècles de la République ont été les pires pour Rome, comme pour le reste du monde $»^{22}$; et c'est probablement dans cette perspective que les expéditions de Bretagne notamment apparaissent sous un jour bien différent que dans le De bello gallico, mais plus généralement encore toute la campagne de Gaule, présentée pour les Romains comme une succession de victoires, mais aussi de revers, et dont Orose conclut le récit en déplorant les malheurs d'un pays tombé sous les coups d'un ennemi impitoyable, quoiqu'un jour destiné à subir le même sort.

À côté cependant de la tradition représentée par Orose et par Bède, qui étant donné l'énorme diffusion de ces deux textes a sans aucun doute fortement orienté la lecture médiévale des expéditions bretonnes de César, il existe au moins une autre version des faits rapportée dans un texte qui a été assez bien diffusé lui aussi : il s'agit de l'Historia Brittonum anonyme, longtemps attribuée au mystérieux Nennius, et dont la plus ancienne version pourrait remonter aux environs de $800^{23}$. Je me

\footnotetext{
${ }^{20}$ M.-P. Arnaud-Lindet note à ce propos (op. cit., p. XXVI, note 57) : «Orose a parcouru rapidement les huit livres du De bello gallico en y opérant des choix ponctuels; le manque de réflexion et la hâte de l'abréviateur se trahissent particulièrement dans le traitement de l'information contenue dans le livre VII de César, pourtant le plus important ».

${ }^{21}$ Quant à l'erreur concernant la date des deux expéditions (60 avant Jésus-Christ), elle est aussi à imputer à Orose, qui se trompe de deux ans à propos du consulat de César et de Bibulus; erreur redoublée cependant par Bède ou sa source directe, puisque cette précision chronologique, donnée chez Orose avant le récit de la conquête de la Gaule (livre VI, 7, 1), apparaît dans l'Histoire ecclésiastique sortie de son contexte.

${ }^{22}$ M.-P. Arnaud-Lindet, éd. cit., p. XXXII.

${ }^{23}$ Il existe de ce texte à la tradition manuscrite particulièrement complexe plusieurs versions, qui n'ont pas toutes été éditées; D.N. Dumville avait naguère ce projet, mais à notre
} 
permets là aussi de citer le texte en intégralité, d'après la traduction qu'en propose C. Kerboul-Vilhon (§ 19-20) :

Les Romains donc, tandis qu'ils dominaient la terre entière, envoyèrent des légats aux Bretons pour obtenir d'eux des otages ainsi que le tribut, comme ils le recevaient de tous les continents et de toutes les îles. Mais les Bretons, à l'esprit indépendant et fier, n'obtempérèrent pas à la requête de l'ambassade romaine. Alors Jules César, le premier à avoir acquis et exercé seul le pouvoir, fut profondément irrité. Il vint en Bretagne avec 60 navires et toucha l'embouchure de la Tamise, où ses navires subirent un naufrage, tandis que lui-même combattait contre Dolobellus, proconsul du roi de Bretagne, nommé Bellinus et fils de Minocanus, qui avait occupé toutes les îles de la mer Tyrrhénienne. Jules César s'en retourna sans avoir remporté la victoire, ses soldats massacrés et ses navires endommagés.

Une nouvelle fois, trois ans après, il revint avec une forte armée et 300 navires de guerre ; il parvint à l'embouchure du fleuve appelé Tamise. Là, il livra bataille et ses chevaux et ses soldats moururent en grand nombre : en effet, le proconsul dont j'ai parlé plus haut avait disposé dans le gué du fleuve des pieux en fer et des «semences de guerre »; ces pièges invisibles mirent les soldats romains dans une situation critique et ils partirent, cette fois-là encore, sans avoir imposé de traité de paix. Une troisième bataille eut lieu à côté de l'endroit appelé Trinovantum, et Jules César y gagna la soumission du peuple breton, 47 ans avant la naissance du Christ, 5215 ans depuis le commencement du monde. Jules César fut donc le premier à venir en Bretagne et à dominer ce pays et son peuple; en son honneur les Romains décidèrent que le mois de Quintilis devait dorénavant s'appeler Julius.

Il semble, à la lecture de ce texte, que l'on ait affaire à une version notablement différente des faits, par l'organisation aussi bien que par les éléments qu'elle contient. D'abord le récit de l'Historia Brittonum tend dans son organisation, plutôt que deux expéditions, à distinguer trois batailles, la dernière seule étant donnée, et uniquement de façon implicite, comme une victoire romaine ayant entraîné la soumission de la Bretagne. En outre le récit, clos sur lui-même, se trouve en quelque sorte doté d'une introduction et d'une conclusion, la première évoquant l'envoi d'ambassadeurs romains - détail entièrement absent chez Orose ou Bède, mais qui se trouve justement chez César(IV, 21) - et la seconde soulignant l'honneur fait au général romain pour avoir été le premier à aller en Bretagne, précision certainement empruntée, comme le montre E. Faral, à la Chronique de saint Jérôme, mais qui n'en rappelle pas moins étrangement le De bello gallico IV, 38. Ensuite les divergences par rapport à Orose ou Bède s'avèrent relativement

connaissance un seul volume est paru sur les huit que devait compter son édition de toutes les versions de l'Historia Brittonum (The Historia Brittonum. The Vatican Recension, Cambridge, Brewer, 1985). À défaut on peut toujours se reporter pour le texte latin à E. Faral (La Légende arthurienne. Les plus anciens textes, Paris, Champion, 1929, tome trois), F. Lot (Nennius et l'Historia Brittonum. Étude critique suivie d'une édition des diverses versions de ce texte, Paris, Champion, 1934) ou J. Morris (Nennius, British History and The Welsh Annals, éd. et trad. J. Morris, Londres et Chichester, Phillimore, 1980), et pour une traduction française à C. Kerboul-Vilhon (Sautron, Éditions du Pontig, 1999). 
nombreuses: divergences de dates (trois ans entre les deux expéditions), de lieux (deux fois la Tamise, et la ville de Trinovantum, tirée certainement du peuple des Trinovantes), de chiffres (60 et 300 au lieu de 80 et 600 navires), enfin de personnages (disparition de Cassobellaunus, remplacé par le roi de Bretagne Bellinus et son proconsul Dolobellus). Selon E. Faral, et H. Nearing à sa suite, mais aussi F. Lot ${ }^{24}$, il apparaît hors de doute que cette version des événements, avec toutes ses innovations, est directement issue d'Orose, y compris le nom du roi de Bretagne et celui de son père, qui seraient empruntés par erreur à un autre passage des Histoires, évoquant l'expédition en Bretagne de Caligula, et non de César. À défaut d'entreprendre une nouvelle enquête sur ce sujet, je me permettrais d'exprimer quelques doutes sur ce point, et d'apporter quelques réflexions complémentaires. En premier lieu, je ne crois pas qu'on puisse être aussi affirmatif concernant les sources de l'Historia Brittonum: s'il est probable que l'auteur de ce texte a utilisé Orose, il n'est pas moins probable qu'il ait eu recours à d'autres sources présentant une version insulaire des faits. Mais ce n'est pas là le plus notable : l'essentiel en effet est qu'on constate, par rapport à Orose ou Bède, un changement d'orientation important, puisque le récit n'est plus seulement défavorable aux Romains, mais devient favorable aux Bretons «à l'esprit indépendant et fier », et qui battent à deux reprises les Romains, avant de se soumettre, on ne sait d'ailleurs comment. Ainsi, soit que l'auteur ait disposé d'une version insulaire des faits, soit qu'il se soit contenté de déformer sciemment le texte d'Orose dans un sens favorable aux Bretons, soit encore qu'il ait fait les deux à la fois, le résultat est le même : c'est la mise en place, dès le $\mathrm{IX}^{\mathrm{e}}$ siècle, d'une tradition insulaire, d'une autre version des premiers pas romains en Bretagne, qu'elle soit résurgence de traditions autochtones plus anciennes ou création de toutes pièces.

Dès le haut Moyen Âge, il existe donc au moins deux traditions partiellement différentes et plus ou moins indépendantes, mais également défavorables aux Romains, concernant leurs premiers pas en Bretagne, qui vont l'une et l'autre s'imposer au détriment du récit effectué par César lui-même, et ce au moins jusqu'au XIII ${ }^{\mathrm{e}}$ siècle. En effet, les textes qui véhiculent ces deux traditions, qu'il s'agisse des Histoires d'Orose (qui, parce qu'elles constituent un abrégé pratique d'histoire universelle, qui plus est dans une perspective chrétienne, ont connu un succès phénoménal depuis la fin de l'Antiquité et pendant tout le Moyen Âge : on en connaît au moins 275 manuscrits), de l'Histoire ecclésiastique de Bède (autre bestseller médiéval, dont on conserve plus de 160 témoins), ou même de l'Historia Brittonum (35 manuscrits, selon J. Morris), ces textes donc ont tous connu une diffusion bien plus importante que ceux de César (environ une trentaine de manuscrits entre le $\mathrm{IX}^{\mathrm{e}}$ et le XII ${ }^{\mathrm{e}}$ siècle, selon le relevé de B. Munk Olsen ${ }^{25}$ ). Il est probable en outre, chose surprenante, qu'il ne s'agisse pas tant, en tout cas pour Bède, mais peut-être aussi pour l'Historia Brittonum, d'une tradition défavorable aux Romains parce qu'elle serait spécifiquement bretonne, mais tout simplement parce qu'elle reproduit les gauchissements dont Orose est le premier responsable.

\footnotetext{
${ }^{24}$ Voir F. Lot, op. cit., p. 54-55 et E. Faral, op. cit., tome un, p. 86-92, ce dernier repris à peu près tel quel par H. Nearing dans son art. cit., p. 896-897.

${ }^{25}$ B. Munk Olsen, L'Étude des auteurs classiques latins aux XI et XII ${ }^{e}$ siècles, CNRS, 19821987.
} 


\section{Le XII siècle : Henri de Huntingdon et Geoffroy de Monmouth}

Aux $\mathrm{XI}^{\mathrm{e}}$ et $\mathrm{XII}^{\mathrm{e}}$ siècles, d'abord en latin, puis en français, l'historiographie anglaise, ou plus précisément normande, connaît un développement sans précédent. Parmi les nombreux historiens écrivant en latin, j'en retiendrai deux qui dans leur histoire d'Angleterre reviennent sur les expéditions césariennes, et fournissent deux exemples de traitements assez différents appliqués à la double tradition transmise par Bède d'un côté et par l'Historia Brittonum de l'autre : Henri de Huntingdon et Geoffroy de Monmouth.

Henri de Huntingdon, donc, publie une première version de son Historia Anglorum en 1129, où il est question des expéditions césariennes (livre I, $\S 12-15)^{26}$. La source première de ce récit, c'est de toute évidence Bède, suivi de très près et textuellement. Néanmoins certains indices montrent que Henri a dû également avoir recours à l'Historia Brittonum : ainsi la distinction de trois batailles différentes, qui tend à recouvrir celle de deux expéditions, les noms de Dolobellus et de Belinus, ou encore le nouveau nom donné au mois de juillet en l'honneur de César. Le plus notable cependant n'est pas l'utilisation conjointe de ces deux traditions, mais l'orientation nouvelle que tend à leur donner Henri, grâce à un certain nombre d'ajouts de son cru, tels le discours d'exhortation de César à ses troupes ( $§ 13)$, les réflexions sur la supériorité militaire des Romains à l'occasion de la troisième bataille ( apparuit virtus Romana dum sagatius ordinati cautius pugnant, obstinatius perseverant», § 14), ou encore l'«autentica laus Cesaris ex Solino» sur laquelle se clôt le récit, en réalité empruntée à Paul Diacre ${ }^{27}$. Ces ajouts montrent clairement l'intention, à partir d'une double tradition peu favorable aux Romains, de redonner au moins partiellement à César et aux siens ce qui leur appartient.

Geoffroy de Monmouth, de son côté, dans son Historia regum Britannice $(\text { vers } 1138)^{28}$, certainement à partir des mêmes sources - l'Historia Brittonum, mais aussi Bède et Henri de Huntingdon lui-même ${ }^{29}$, et peut-être également des sources (orales) indigènes ${ }^{30}$, mais sans oublier surtout une bonne part d'invention personnelle -, arrive à un résultat tout différent. En fait on atteint, avec Geoffroy, à

\footnotetext{
${ }^{26}$ Henri de Huntingdon, Historia Anglorum, éd. et trad. D. Greenway, Oxford, Clarendon, 1996

${ }^{27}$ Paul Diacre, Historia romana (éd. A. Crivellucci, Rome, 1914), VI, 25. On notera en passant que la campagne bretonne de César se trouve fugitivement évoquée dans le même ouvrage, p. 91.

${ }^{28}$ Nous citons le texte d'après la traduction de L. Mathey-Maille, Histoire des rois de Bretagne, Paris, Les Belles Lettres, 1992. Le texte latin traduit par L. Mathey-Maille se trouve dans The Historia Regum Britannia of Geoffrey of Monmouth, éd. N. Wright, tome un, Bern, Burgerbibliothek, ms. 568, Cambridge, Brewer, 1984.

${ }^{29}$ Voir notamment les commentaires d'E. Faral dans La Légende arthurienne, tome deux, p. 142-158.

${ }^{30}$ Voir sur ce point C. Sterckx, «Les sources du récit de la conquête romaine chez Geoffrey de Monmouth et Robert Wace », Le Roman de Brut entre mythe et histoire, éd. C. Letellier et D. Hüe, Orléans, Paradigme, 2003, p. 89-100. Et toujours à propos de la campagne bretonne, on pourra se reporter à un autre article du même auteur, "De Cassivellaunos à Caswallon », Studia celtica, 32, 1998, p. 95-114.
} 
une sorte de point d'aboutissement, à la fois parce qu'il s'agit depuis César luimême du récit le plus développé ( $\$ 54-63$, soit treize pages dans la traduction de L. Mathey-Maille), le plus circonstancié - mais qui devient véritablement romanesque - concernant les premiers pas des Romains en Bretagne, et en même temps parce qu'il s'agit de la version de ces événements de loin la plus défavorable à César, avec pour résultat un renversement total de perspective vis-à-vis du $D e$ bello gallico. La source principalement suivie par Geoffroy est visiblement l'Historia Brittonum, dont il adopte l'organisation temporelle en trois batailles et reprend la matière, en la développant toutefois assez considérablement. D'abord une introduction ( $\$$ 54-55) reprend l'idée d'une ambassade romaine envoyée en Bretagne, mais elle est ici remplacée par un message de César au roi Cassibellan et exigeant soumission et tribut de la part des Bretons, Geoffroy rappelant à cette occasion - autre idée empruntée à l'Historia Brittonum - que Bretons et Romains partagent la même origine en descendant tous des Troyens. D'emblée se manifeste le double mouvement qui caractérise tout le récit de Geoffroy, visant à élever les Bretons tout en rabaissant les Romains. Pour la première expédition romaine et la première bataille ( $§$ 56-57), Geoffroy s'appuie sur les données de l'Historia Brittonum, qu'il romance allègrement, en particulier dans la description du combat entre Nennius, le frère de Cassibellan (mais peut-être faut-il y voir une reconnaissance de dette vis-à-vis de son devancier?), et Jules César lui-même, où ce dernier perd sa fameuse épée ; en tout cas, dans la bataille comme dans le combat des deux chefs, ce sont les Bretons qui l'emportent, d'où le départ des Romains. Le $\S 58$ prolonge les effets de cette première défaite, avec la fuite humiliante de Bretagne et la révolte des Gaulois devant qui César est obligé de faire le dos rond, et fait transition avec la seconde expédition, qui apparaît uniquement motivée par un désir de vengeance. Puis c'est la même vision des choses qui est mise en œuvre lors de la seconde expédition et de la seconde bataille: Geoffroy brode à partir de l'Historia Brittonum, en rabaissant les Romains pour mieux glorifier les Bretons. Enfin le récit ( $\S 61-63$ ) se complique un peu pour la troisième bataille, dans la mesure où il faut justifier la victoire définitive des Romains, qui n'est guère plus qu'une demie victoire chez Geoffroy, puisque César ne vainc pas Cassibellan mais fait seulement la paix avec lui ; c'est de là que viennent les développements autour du personnage d'Androgeus, dont le comportement est donné pour responsable de la défaite bretonne, puisqu'il a appelé à l'aide César dans un conflit personnel l'opposant à Cassibellan; et tandis que Cassibellan assiégé résiste toujours à l'envahisseur, Geoffroy laisse éclater ses sympathies en s'exclamant: «Oh! Quel peuple admirable était en ce temps-là le peuple breton qui, par deux fois, avait mis en fuite le maître de l'univers! Celui à qui la terre entière avait dû céder, les Bretons, même battus, lui résistaient, prêts à mourir pour la patrie et pour la liberté. C'est en leur honneur que Lucain écrivit ce vers sur César: [César] fuit avec effroi les Bretons qu'il cherchait à combattre» (\$ 62). Constant dans son projet, Geoffroy n'est donc pas seulement capable de broder sur l'Historia Brittonum pour louer les Bretons, mais aussi de convoquer à bon escient une autorité telle que Lucain pour ajouter encore à sa critique de César. 


\section{Conclusion}

Geoffroy présente donc une sorte de point d'aboutissement dans notre parcours. Sa version des faits est, de loin, la plus défavorable de toutes - à tel point d'ailleurs, comme on l'a dit plus haut, qu'elle a suscité des réactions, qu'il s'agisse des critiques que lui adresse un Guillaume de Newburgh dans le prologue de son Historia rerum anglicarum ou du portrait flatteur de César qu'ajoute Wace à son adaptation du texte de Geoffroy. Cette version est la plus défavorable de toute car elle croise en fait deux traditions déjà bien peu favorables à César, dont il est difficile de juger si elles sont indépendantes ou non: la première, très largement répandue grâce au succès d'Orose puis de Bède au Moyen Âge, remonte en dernier ressort à César, mais un César résumé jusqu'à en être méconnaissable, la faute à Orose et au projet de ses Histoires parfois dites contre les païens; la seconde, également bien répandue, est celle de l'Historia Brittonum, qui représente peut-être une tradition plus autochtone, et donc plus directement favorable aux Bretons, même s'il est possible qu'elle s'appuie aussi sur le même texte d'Orose. Enfin, sur ce terreau déjà propice, Geoffroy n'hésite aucunement à faire pousser des fleurs de son invention, pour mieux rehausser les Bretons en abaissant les Romains.

Aussi atypique, aussi outrée soit-elle, la version de Geoffroy n'en a pas moins bénéficié d'une diffusion exceptionnelle: en effet, on connaît pas moins de 215 manuscrits $^{31}$ de l'Historia regum Britannice, ce qui en fait un des textes historiques les plus lus du Moyen Âge ${ }^{32}$. Un tel succès n'aura pas été sans nuire fortement à l'image de César, particulièrement dans le milieu normand qui a vu naître ce texte. Comment alors, dans ce même milieu, imaginer de commanditer une adaptation de La Guerre civile, de faire un roman avec César pour héros ou pour personnage principal, lui qui fut l'ennemi des Bretons, et qui finalement - comme le rappelle justement Pompée dans le poème de Lucain - n'avait pas même été capable de les vaincre?

Silvère Menegaldo Université d'Orléans

\footnotetext{
${ }^{31}$ Voir J. Crick, The Historia regum Britanniae of Geoffrey of Monmouth. III. A Summary Catalogue of the Manuscripts, Cambridge, Brewer, 1989.

${ }^{32}$ Voir le tableau fourni par B. Guenée dans Histoire et culture historique dans l'Occident médiéval, Paris, Aubier, 1980, p. 250-252.
} 